Pacific Journal of Mathematics

ON PRODUCT BASES 


\title{
ON PRODUCT BASES
}

\author{
JohN C. MoRgan II
}

\begin{abstract}
Starting with the notion of a category base, which is a generalization of a topological space, we investigate cartesian products of category bases. Extension are obtained of several classical topological theorems concerning Baire category in product spaces.
\end{abstract}

In $\S 1$ we formulate the basic concepts and state relevant theorems which were established earlier. In $\S 2$ we give conditions under which the cartesian product of two category bases is also a category base. General properties of product bases are then presented in $\S 3$. Utilizing the notion of a separable category base, discussed in $\S 4$, we establish generalizations of results of Kuratowski and Ulam, Sikorski, and Oxtoby on cartesian products (cf. [2], [10], [11]). Even in the context of topological spaces the theorems given are more general than the previous results.

1. Preliminaries. In this section we review the basic concepts and result which are pertinent to this article. We have also added several examples to clarify the ideas. For proofs of the main theorems see [4], [5], [8]. For other related results see [6], [7], [9].

Definition. A pair $(X, \mathscr{C})$, where $\mathscr{C}$ is a family of subsets of a nonempty set $X$, is called a category base if the nonempty sets in $\mathscr{C}$, called regions, satisfy the following axioms:

1. Every point of $X$ belongs to some region; i.e., $X=\mathbf{U} \mathscr{C}$.

2. Let $A$ be a region and let $\mathscr{D}$ be any nonempty family of disjoint regions which has power less than the power of $\mathscr{C}$.

(a) If $A \cap(\bigcup \mathscr{D})$ contains a region then there is a region $D \in$ $\mathscr{D}$ such that $A \cap D$ contains a region.

(b) If $A \cap(\bigcup \mathscr{D})$ contains no region then there is a region $B \subset A$ which is disjoint from every region in $\mathscr{D}$.

Among the examples of category bases are the following.

EXAMPLE 1A. The family of all complements of finite subsets of an uncountable set.

EXAMPLE 1B. Every topology.

Example 1C. The family of all measurable sets of positive 
measure in a $\sigma$-finite measure space.

EXAMPLE 1D. The family of all perfect sets in a complete separable metric space with no isolated points. It is the inclusion of this example which led to the cardinality restriction in Axiom 2.

With respect to a given category base $(X, \mathscr{C})$ we define the generalized Baire category concepts for subsets of $X$.

DEFinition. A set $S$ is singular if every region contains a subregion which is disjoint from $S$. A countable union of singular sets is called meager set. A set which is not a meager set is called an abundant set. An abundant set whose complement is a meager set is called a comeager set.

Notation. The family of all sets which are meager with respect to a given category base $(X, \mathscr{C})$ will be denoted by $\mathfrak{M}(\mathscr{C})$.

THEOREM 1.1. The intersection of any two regions either contains a region or it is a singular set.

EXAMPLE 1E. If $\mathscr{C}$ is the family of all complements of finite subsets of an uncountable set then the singular sets, meager sets, and abundant sets coincide with the finite, countable, and uncountable sets, respectively.

EXAMPLE 1F. If $(X, \mathscr{C})$ is a topology then the singular, meager, and abundant sets are the nowhere dense sets, sets of the first category, and the sets of the second category, respectively.

ExAMPLe 1G. Let $\mathscr{C}$ denote the family of all measurable sets of positive measure in a $\sigma$-finite measure space $(X, \mathscr{A}, \mu)$ and let $\bar{\mu}$ denote the completion of $\mu$. The families of singular sets and meager sets with respect to $\mathscr{C}$ are identical and coincide with the sets of $\bar{\mu}$-measure zero. The abundant sets coincide with the sets of positive $\bar{\mu}$-outer measure.

EXAMPLE $1 \mathrm{H}$. If $\mathscr{C}$ is the family of all perfect sets in a complete separable metric space with no isolated points then the families of singular sets and of meager sets are identical and coincide with the sets having Marczewski's property $\left(s_{2}^{0}\right)$ (see [12]).

THEOREM 1.2. The family of all singular sets forms an ideal and the family of all meager sets forms a $\sigma$-ideal. 
Definition. A set $S$ is meager (abundant) in a region $A$ if $S \cap A$ is meager (abundant). A set is abundant everywhere in a region $A$ if it is abundant in every subregion of $A$. A set is abundant everywhere if it is abundant in every region.

THEOREM 1.3. If $S$ is abundant everywhere in a region $A$ and $A$ is abundant everywhere in a region $B$ then $S$ is abundant everywhere in $B$.

The most important theorem is the following generalization of the Banach Category Theorem.

Fundamental Theorem. Every abundant set is abundant everywhere in some region.

Corollary 1.4. If a set is abundant in a region $A$ then it is abundant everywhere in some subregion of $A$.

In topology, a set $S$ is defined to be locally of the first category at a point $x$ if there is an open set $G$ containing $x$ such that $S \cap G$ is of the first category. This suggests the general definition that a set $S$ be locally meager at a point $x$ if there is a region $A$ containing $x$ such that $S \cap A$ is a meager set. This definition however is not satisfactory.

For example, if $\mathscr{C}$ is the family of all bounded, closed intervals on the real line then, according to this general definition, the closed unit interval $[0,1]$ would be locally $\mathscr{C}$-meager at the number 1 , while the same set would not be locally $\mathscr{D}$-meager at the number 1 for the category base $\mathscr{D}$ consisting of all bounded, open intervals.

The definition which we have adopted is, in fact, equivalent to the above topological definition.

Definition. A set $S$ is locally meager at a point $x \in X$ if in every region $A$ containing $x$ there is a subregion $B$ containing $x$ such that $S \cap B$ is a meager set.

THeOREM 1.5. A necessary and sufficient condition that a set be meager is that it be locally meager at every point of $X$.

Definition. A set $S$ has the Baire property if every region $A$ has a subregion $B$ in which either $S$ or $X-S$ is a meager set. Restated, $S$ has the Baire property if there is no region in which both $S$ and its complement are abundant everywhere. 
Notation. The family of sets which have the Baire property with respect to $(X, \mathscr{C})$ will be denoted by $\mathfrak{B}(\mathscr{C})$.

EXAMPLE 1I. If $(X, \mathscr{C})$ is a topology then $\mathfrak{B}(\mathscr{C})$ is the family of sets which have the Baire property in the classical sense.

ExAMPLE 1J. If $(X, \mathscr{A}, \mu)$ is a $\sigma$-finite measure space and $\mathscr{C}$ is the family of all sets in $\mathscr{A}$ of positive measure then $\mathfrak{B}(\mathscr{C})$ is the family of all sets measurable with respect to the completion $\bar{\mu}$ of the measure $\mu$.

EXAMPLE 1K. If $X$ is a complete separable metric space with no isolated points and $\mathscr{C}$ is the family of all perfect sets then $\mathfrak{B}(\mathscr{C})$ is the family of all Marczewski sets (i.e., the sets having the property (s) of Marczewski [12]).

THEOREM 1.6. The sets which have the Baire property form a $\sigma$-field which contains all regions and all meager sets.

THEOREM 1.7. If $S$ has the Baire property and is abundant every-where in a region $A$ then $A-S$ is a meager set.

THEOREM 1.8. If $(X, \mathscr{C})$ satisfies CCC (the countable chain condition) then a set $S$ has the Baire property if and only if $S$ is representable as the union of two sets $P, R$, where $P$ is a $\mathscr{C}_{\sigma o}$-set and $R$ is a meager set.

Corollary 1.9. If $(X, \mathscr{C})$ satisfies CCC then $\mathfrak{B}(\mathscr{C})$ is the smallest $\sigma$-field containing all regions and all meager sets.

Definition. Two category bases $(X, \mathscr{C})$ and $(X, \mathscr{D})$ are called equivalent if $\mathfrak{M}(\mathscr{C})=\mathfrak{M}(\mathscr{D})$ and $\mathfrak{B}(\mathscr{C})=\mathfrak{B}(\mathscr{D})$.

THEOREM 1.10. If $(X, \mathscr{C})$ and $(X, \mathscr{D})$ are category bases such that each $\mathscr{C}$-region contains a $\mathscr{D}$-region and, conversely, each $\mathscr{D}$ region contains a $\mathscr{C}$-region, then $(X, \mathscr{C})$ is equivalent to $(X, \mathscr{D})$.

EXAMPLE 1L. If $X$ is the real line then the following families of subsets of $X$ are all equivalent category bases: all open sets in the usual topology, all open intervals, all closed intervals, and all open sets in the lower-limit topology (i.e., all sets which are unions of intervals of the form $[a, b)$ ).

EXAMPLE 1M. If $X$ is an uncountable set then the cofinite 
topology consisting of $\phi, X$, and all complements of finite subsets of $X$ is equivalent to the cocountable topology consisting of $\phi, X$, and all complements of countable subsets of $X$. By means of Corollary 1.9 it is seen that the sets having the Baire property are the sets $S$ such that either $S$ or $X-S$ is countable.

ExAMPLE $1 \mathrm{~N}$. Let $(X, \mathscr{C})$ be a topology. A subfamily $\mathscr{B}$ of $\mathscr{C}$ is called a topological base for $\mathscr{C}$ if every set in $\mathscr{C}$ is a union of members of $\mathscr{B}$. If $\mathscr{B}$ is a topological base for $\mathscr{C}$ then $(X, \mathscr{B})$ is a category base which is equivalent to $(X, \mathscr{C})$.

ExAmple 10. Let $(X, \mathscr{C})$ be a topology. Oxtoby has defined in [10] a family $\mathscr{B}$ of nonempty sets in $\mathscr{C}$ to be a pseudo-base if every nonempty set in $\mathscr{C}$ contains at least one member of $\mathscr{B}$. If $\mathscr{B}$ is a pseudo-base for $\mathscr{C}$ then $(X, \mathscr{B} \cup\{X\})$ is a category base which is equivalent to $(X, \mathscr{C})$.

We shall say a topology $(X, \mathscr{C})$ has an equivalent pseudo-base $\mathscr{B}$ if there is a topology $(X, \mathscr{D})$ such that $\mathscr{B}$ is a pseudo-base for $(X, \mathscr{D})$ and $(X, \mathscr{D})$ is equivalent to $(X, \mathscr{C})$. Alternatively, $(X, \mathscr{C})$ has an equivalent pseudo-base $\mathscr{B}$ if the category base $(X, \mathscr{B} \cup\{X\})$ is equivalent to $(X, \mathscr{C})$.

EXAMPLE 1P. In $n$-dimensional Euclidean space the category base of all Lebesgue measurable sets of positive measure is equivalent to the category base of all closed sets of positive measure and is also equivalent to the category base of all perfect sets which have positive Lebesgue measure in every neighborhood of each of their points.

EXAMPLE 1Q. In a complete separable metric space with no isolated points the category base of all perfect sets is equivalent to the category base of all uncountable Borel sets.

THEOREM 1.11. Let $(X, \mathscr{C})$ be a category base, let $A$ be a region in $\mathscr{C}$, and let

$$
\mathscr{C}_{A}=\{B \in \mathscr{C}: B \subset A\} .
$$

Then $\left(A, \mathscr{C}_{A}\right)$ is a category base, called the relativization of $\mathscr{C}$ to A. Moreover, for every set $S \subset A$ we have

(i) $S$ is $\mathscr{C}_{A}$-singular if and only if $S$ is $\mathscr{C}$-singular.

(ii) $S$ is $\mathscr{C}_{A}$-meager if and only if $S$ is $\mathscr{C}$-meager.

(iii) $S$ has the Baire property with respect to $\left(A, \mathscr{C}_{A}\right)$ if and only if $S$ has the Baire property with respect to $(X, \mathscr{C})$. 
Definition. Let $X$ denote a complete separable metric space with no isolated points. A category base $(X, \mathscr{C})$ consisting of perfect sets is called a perfect base if it satisfies the condition

$(\dagger)$ for every region $A$ and every point $x \in A$ there is a descending sequence $\left\langle A_{n}\right\rangle_{n=1}^{\infty}$ of regions such that $x \in A_{n}, A_{n} \subset A$, and $\operatorname{diam}\left(A_{n}\right) \leqq 1 / n$ for each $n$.

THEOREM 1.12. If $(X, \mathscr{C})$ is a perfect category base then every abundant set contains a set which does not have the Baire property.

CoRollary 1.13. If $(X, \mathscr{C})$ and $(X, \mathscr{D})$ are non-equivalent perfect category bases and $\mathscr{C} \subset \mathscr{D}$ then there exists a set which has the Baire property with respect to $\mathscr{C}$ but which does not have the Baire property with respect to $\mathscr{D}$. In fact, there exists a $\mathscr{C}$-singular set which does not have the Baire property with respect to $\mathscr{D}$.

2. Definition and examples. We first show that if $(X, \mathscr{C})$ and $(Y, \mathscr{D})$ are category bases then $(X \times Y, \mathscr{C} \times \mathscr{D})$ is not necessarily a category base.

Example 2A. Let $X$ denote the closed unit interval [0,1], let $Q$ denote the set of all rational numbers, let $C$ denote the Cantor set, and let

$$
\begin{aligned}
\mathscr{F}= & \{[0,1],[0,1 / 3],[2 / 3,1],[0,1 / 9],[2 / 9,1 / 3], \\
& {[2 / 3,7 / 9],[8 / 9,1], \cdots\} }
\end{aligned}
$$

be the family of closed intervals used in the construction of $C$. Note that any two intervals in $\mathscr{I}$ are either disjoint or one interval is contained in the other. We shall show the countable family

$$
\mathscr{C}=\{[a, b]: 0 \leqq a<b \leqq 1 \text { and } a, b \in Q\} \cup\{C \cap I: I \in \mathscr{I}\}
$$

is a category base.

Axiom 1 is obviously satisfied. Suppose then that $A \in \mathscr{C}$ and $\left\{D_{1}, \cdots, D_{n}\right\}$ is a nonempty, finite family of disjoint sets in $\mathscr{C}$.

If $A \cap\left(\mathbf{U}_{i=1}^{n} D_{i}\right)$ contains a set in $\mathscr{C}$ then there is an index $i$ such that $A \cap D_{i}$ is uncountable. If $A$ and $D_{i}$ are either both closed intervals with rational endpoints or both sets of the form $C \cap I$ where $I \in \mathscr{F}$, then their intersection will also be of the same common form and hence will be a set in $\mathscr{C}$. On the other hand, if $A=[a, b]$ and $D_{i}=C \cap I$ for some $I \in \mathscr{A}$, or vice versa, then $A \cap D_{i}$ will contain a set of the form $C \cap J$ for some $J \in \mathscr{F}$. Thus Axiom 2a is verified.

Suppose now that $A \cap\left(\bigcup_{i=1}^{n} D_{i}\right)$ contains no set in $\mathscr{C}$. For each $i$, the set $A \cap D_{i}$ will then be countable. This implies that each of 
the sets $A \cap D_{i}$ contains at most two points. There will therefore be a set $B \in \mathscr{C}$ which is contained in $A$ and is disjoint from each of the sets $D_{i}$. Thus Axiom $2 \mathrm{~b}$ is also verified.

Let $Y=[0,1]$ and let

$$
\mathscr{D}=\{[a, b]: 0 \leqq a<b \leqq 1\} .
$$

As is easily verified, $\mathscr{D}$ is a category base satisfying CCC. The product family $\mathscr{C} \times \mathscr{D}$ also satisfies CCC, but it is not a category base.

For, let $A_{0}=C$, let $A_{1}=[1 / 3,2 / 3], A_{2}=[1 / 9,2 / 9], A_{3}=[7 / 9,8 / 9], \cdots$ be the sequence of intervals both of whose endpoints alone belong to $C$, and let $B_{n}=[0,1]$ for each $n=0,1,2, \cdots$. The family $\left\{A_{n} \times B_{n}: n=1,2, \cdots\right\}$ is nonempty, disjoint subfamily of $\mathscr{C} \times \mathscr{D}$ which has power less than the power of $\mathscr{C} \times \mathscr{D}$, the set $\left(A_{0} \times B_{0}\right) \cap$ $\left[\bigcup_{n=1}^{\infty}\left(A_{n} \times B_{n}\right)\right]$ contains no set in $\mathscr{C} \times \mathscr{D}$, and there is no set in $\mathscr{C} \times \mathscr{D}$ which is contained in $\left(A_{0} \times B_{0}\right)-\left[\bigcup_{n=1}^{\infty}\left(A_{n} \times B_{n}\right)\right]$. Axiom $2 \mathrm{~b}$ is therefore not satisfied.

DEFinition. If $(X, \mathscr{C})$ and $(Y, \mathscr{D})$ are category bases such that $(X \times Y, \mathscr{C} \times \mathscr{D})$ is a category base, then $(X \times Y, \mathscr{C} \times \mathscr{D})$ is called a product base.

In order to formulate a general theorem concerning the existence of product bases, we introduce the following property.

Definition. Let $(X, \mathscr{C})$ be a category base. For each cardinal number $\mathfrak{m}$ we define the property

$P(\mathfrak{m})$ : If $\mathscr{C}$ is a family of $\mathscr{C}$-regions which has power $<\mathfrak{m}$ and $C$ is a $\mathscr{C}$-region such that $C \cap M$ contains no $\mathscr{C}$-region for every $M \varepsilon \mathscr{A}$, then there exists a $\mathscr{C}$-region $E \subset C$ which is disjoint from every region in $\mathscr{M}$.

Note that in the case that $\mathscr{C}$ has power $m$, Axiom $2 b$ is a consequence of the property $P(\mathfrak{m})$.

TheOREM 2.1. If $(X, \mathscr{C})$ and $(Y, \mathscr{D})$ are category bases, $\mathscr{C} \times \mathscr{D}$ has power $\mathfrak{m}$, and both $\mathscr{C}$ and $\mathscr{D}$ have the property $P(\mathfrak{m})$, then $(X \times Y, \mathscr{C} \times \mathscr{D})$ is a category base.

Proof. Axiom 1 being obviously true, we have only to verify Axiom 2.

Let $\left\{A_{\alpha} \times B_{\alpha}: \alpha \in I\right\}$ be a nonempty family of disjoint $(\mathscr{C} \times \mathscr{D})$ - 
regions which has power less than the power of $\mathscr{C} \times \mathscr{D}$ (hence the index set $I$ has power $<\mathfrak{m})$ and let $A \times B$ be any $(\mathscr{C} \times \mathscr{D})$-region. Define

$$
\begin{aligned}
& J=\left\{\alpha \in I: A \cap A_{\alpha} \text { contains no } \mathscr{C} \text {-region }\right\} \\
& K=\left\{\alpha \in I: B \cap B_{\alpha} \text { contains no } \mathscr{D} \text {-region }\right\}
\end{aligned}
$$

Suppose

$$
(A \times B) \cap\left[\bigcup_{\alpha \in I}\left(A_{\alpha} \times B_{\alpha}\right)\right]=\bigcup_{\alpha \in I}\left[\left(A \cap A_{\alpha}\right) \times\left(B \cap B_{\alpha}\right)\right]
$$

contains a $(\mathscr{C} \times \mathscr{D})$-region $C \times D$. Since $C$ is a subset of $A$, we have $C \cap A_{\alpha}=C \cap\left(A \cap A_{\alpha}\right)$ for every $\alpha \in I$. Hence, for every index $\alpha \in J$, the set $C \cap A_{\alpha}$ contains no $\mathscr{C}$-region. Because $\mathscr{C}$ has property $P(\mathfrak{m})$, there exists a $\mathscr{C}$-region $E \subset C-\bigcup_{\alpha \in J} A_{\alpha}$. Similarly, there is a $\mathscr{D}$-region $F \subset D-\bigcup_{\alpha \in K} B_{\alpha}$. The set $E \times F$ is a $(\mathscr{C} \times \mathscr{D})$-region and we have

$$
E \times F \subset C \times D \subset \bigcup_{\alpha \in I}\left(A_{\alpha} \times B_{\alpha}\right) .
$$

Since $E \times F \neq \varnothing$, there is an index $\alpha_{0} \in I$ such that $(E \times F) \cap$ $\left(A_{\alpha_{0}} \times B_{\alpha_{0}}\right) \neq \varnothing$. In particular, $E \cap A_{\alpha_{0}} \neq \varnothing$ and $F \cap B_{\alpha_{0}} \neq \varnothing$. In view of the definition of $E$ and $F$, these relationships imply $\alpha_{0} \notin J$ and $\alpha_{0} \notin K$. Consequently $A \cap A_{\alpha_{0}}$ contains a $\mathscr{C}$-region and $B \cap B_{\alpha_{0}}$ contains a $\mathscr{D}$-region. We then conclude $(A \times B) \cap\left(A_{\alpha_{0}} \times B_{\alpha_{0}}\right)$ contains a $(\mathscr{C} \times \mathscr{D})$-region and Axiom 2a is thereby verified.

Suppose $(A \times B) \cap\left[\bigcup_{\alpha \in I}\left(A_{\alpha} \times B_{\alpha}\right)\right]$ contains no $(\mathscr{C} \times \mathscr{D})$-region. Then, for every index $\alpha \in I$, either $A \cap A_{\alpha}$ contains no $\mathscr{C}$-region or $B \cap B_{\alpha}$ contains no $\mathscr{D}$-region. Choose a $\mathscr{C}$-region $C \subset A-\bigcup_{\alpha \in J} A_{\alpha}$ and a $\mathscr{D}$-region $D \subset B-\bigcup_{\alpha \in K} B_{\alpha}$. Because $I=J \cup K$, we have

$$
C \times D \subset(A \times B)-\bigcup_{\alpha \in I}\left(A_{\alpha} \times B_{\alpha}\right) .
$$

Therefore, Axiom $2 \mathrm{~b}$ is also verified.

$$
(X \times Y, \mathscr{C} \times \mathscr{D}) \text { is thus a product base . }
$$

THEOREM 2.2. If $(X, \mathscr{C})$ and $(Y, \mathscr{D})$ are category bases, $\mathscr{C} \times$ $\mathscr{D}$ satisfies CCC, and both $\mathscr{C}$ and $\mathscr{D}$ have the property $P\left(\boldsymbol{\aleph}_{1}\right)$, then $(X \times Y, \mathscr{C} \times \mathscr{D})$ is a category base.

Proof. One need only repeat the proof of Theorem 2.1 with $\mathfrak{m}$ replaced by $\aleph_{1}$.

EXAMPLE 2B. In Example 2A, the family $\mathscr{C}$ has the property 
$P\left(\boldsymbol{\aleph}_{0}\right)$, but does not have the property $P\left(\boldsymbol{\aleph}_{1}\right)$, the family $\mathscr{D}$ has the property $P(\mathfrak{m})$ for every cardinal number $\mathfrak{m}$, and $\mathscr{C} \times \mathscr{D}$ has power $2^{x_{0}}$. The family

$$
\mathscr{E}=\{[a, b]: 0 \leqq a<b \leqq 1 \text { and } a, b \in Q\}
$$

is a category base which is equivalent to $\mathscr{D}$ and has the property $P\left(\boldsymbol{\aleph}_{0}\right)$. Since $\mathscr{C} \times \mathscr{E}$ has power $\boldsymbol{\aleph}_{0}$, it follows from Theorem 2.1 that $(X \times Y, \mathscr{C} \times \mathscr{E})$ is a category base.

ExAmple 2C. If $(X, \mathscr{C})$ and $(Y, \mathscr{D})$ are topologies then both $\mathscr{C}$ and $\mathscr{D}$ are category bases which have the property $P(\mathfrak{m})$ for every cardinal number $m$. By Theorem $2.1,(X \times Y, \mathscr{C} \times \mathscr{D})$ is a category base. In general, this product base is not itself a topology, but it is equivalent to Tychonoff's product topology.

EXAMPLE 2D. If $\mathscr{B}$ is a pseudo-base for a topology $(Y, \mathscr{D}), B_{0}$ is a fixed set in $\mathscr{B}$, and

$$
\mathscr{E}=\mathscr{B} \cup\left\{B_{0} \cup(Y-\cup \mathscr{B})\right\}
$$

then $(Y, \mathscr{E})$ is a category base which is equivalent to $(Y, \mathscr{D})$ and has the property $P(\mathfrak{m})$ for every cardinal number $\mathfrak{m}$. Hence, if $(X, \mathscr{C})$ is any topology then $(X \times Y, \mathscr{C} \times \mathscr{E})$ is a product base.

ExAMPLE 2E. If $\mathscr{C}$ and $\mathscr{D}$ are the families of all measurable sets of positive measure in $\sigma$-finite measure spaces $(X, \mathscr{A}, \mu)$ and $(Y, \mathscr{B}, \nu)$, respectively, then $(X, \mathscr{C})$ and $(Y, \mathscr{D})$ are category bases each of which has the property $P\left(\boldsymbol{N}_{1}\right)$ and $\mathscr{C} \times \mathscr{D}$ satisfies CCC. By Theorem $2.2,(X \times Y, \mathscr{C} \times \mathscr{D})$ is a product base.

Example 2F. Let $(X, \mathscr{C})$ and $(Y, \mathscr{D})$ both denote the perfect category base of all perfect linear sets which have positive Lebesgue measure in every neighborhood of each of their points. Using Theorem 2.2 , we see that $(X \times Y, \mathscr{C} \times \mathscr{D})$ is also a perfect, category base. However, although $\mathfrak{B}(\mathscr{C})$ and $\mathfrak{B}(\mathscr{D})$ both coincide with the family of all linear Lebesgue measurable sets, the family $\mathfrak{B}(\mathscr{C} \times \mathscr{D})$ is not the same as the family of all sets measurable with respect to planar Lebesgue measure (i.e., all sets having the Baire property with respect to the perfect, category base $\mathscr{E}$ of all perfect, planar sets which have positive planar Lebesgue measure in every neighborhood of each of their points).

For, assume to the contrary that $\mathfrak{B}(\mathscr{C} \times \mathscr{D})=\mathfrak{B}(\mathscr{E})$. It then follows from Theorem 1.12 that the $(\mathscr{C} \times \mathscr{D})$-meager sets coincide with the sets of planar Lebesgue measure zero. Now, one can establish 
the existence of a planar Lebesgue measurable set $E$ such that both $E$ and its complement intersect every $(\mathscr{C} \times \mathscr{D})$-region in a set of positive, planar Lebesgue measure (cf. [1]). Such a set $E$ does not have the Baire property with respect to $\mathscr{C} \times \mathscr{D}$, since both $E$ and its complement would be everywhere $(\mathscr{E} \times \mathscr{D})$-abundant. Therefore, $\mathfrak{B}(\mathscr{C} \times \mathscr{D}) \neq \mathfrak{B}(\mathscr{C})$.

We conclude from Corollary 1.13 that there exists a set which is $\left(\mathscr{C}^{2} \times \mathscr{D}\right)$-meager, but which is not Lebesgue measurable.

Example 2G. If $X, Y$ are complete, separable metric spaces with no isolated points and $\mathscr{P}(X), \mathscr{P}(Y)$ are the families of all perfect sets in $X, Y$, respectively, then both $\mathscr{P}(X)$ and $\mathscr{P}(Y)$ have the property $P\left(2^{\mathrm{N}_{0}}\right)$. Since $\mathscr{P}(X) \times \mathscr{P}(Y)$ has power $2^{\mathrm{N}_{0}},(X \times Y$, $\mathscr{P}(X) \times \mathscr{P}(Y))$ is a product base. Although the sets having the Baire property with respect to $\mathscr{P}(X)$ and $\mathscr{P}(Y)$ are the Marczewski sets in $X$ and $Y$, respectively, if $X \times Y$ is metrized with the usual Euclidean product metric, the sets which have the Baire property with respect to $\mathscr{P}(X) \times \mathscr{P}(Y)$ do not coincide with the Marczewski sets in $X \times Y$.

For example, suppose $x_{0} \in X$ and $L=\left\{x_{0}\right\} \times Y$. Being a planar perfect set, $L$ is a Marczewski abundant set with respect to the family of all perfect sets in $X \times Y$. By Theorem 1.12, $L$ contains a set $S$ which is not a planar Marczewski set. However, $S$ is obviously a $(\mathscr{P}(X) \times \mathscr{P}(Y))$-singular set and hence has the Baire property with respect to $\mathscr{P}(X) \times \mathscr{P}(Y)$.

The proof of the following theorem is straight-forward.

THEOREM 2.3. If $(X \times Y, \mathscr{C} \times \mathscr{D})$ is a product base and $E$ is any $\mathscr{D}$-region then $\left(X \times E, \mathscr{C} \times \mathscr{D}_{E}\right)$ is also a product base. Moreover for every set $S \subset X \times E$ we have

(i ) $S$ is $\left(\mathscr{C} \times \mathscr{D}_{E}\right)$-singular if and only if $S$ is $(\mathscr{C} \times \mathscr{D})$ singular.

(ii) $S$ is $\left(\mathscr{C} \times \mathscr{D}_{E}\right)$-meager if and only if $S$ is $(\mathscr{C} \times \mathscr{D})$ meager.

(iii) $S$ has the Baire property with respect to $\mathscr{C} \times \mathscr{D}_{E}$ if and only if $S$ has the Baire property with respect to $\mathscr{C} \times \mathscr{D}$.

3. General properties. We assume throughout this section that $(X \times Y, \mathscr{C} \times \mathscr{D})$ is a given product base.

Theorem 3.1. If $S \subset X$ and $T \subset Y$ then $S \times T$ is $(\mathscr{C} \times \mathscr{D})$ singular if and only if $S$ is $\mathscr{C}$-singular or $T$ is $\mathscr{D}$-singular. 
Proof. Suppose $S \times T$ is $(\mathscr{C} \times \mathscr{D})$-singular and $T$ is not $\mathscr{D}$ singular. Then there is a $\mathscr{D}$-region $D$ such that for every $\mathscr{D}$ region $E \subset D$ we have $E \cap T \neq \varnothing$. If $A$ is any $\mathscr{C}$-region then $A \times D$ is a $(\mathscr{C} \times \mathscr{D})$-region and, by hypothesis, there is a $(\mathscr{C} \times \mathscr{D})$-region $B \times E \subset A \times D$ such that

$$
(B \times E) \cap(S \times T)=(B \cap S) \times(E \cap T)=\varnothing .
$$

Since $B \times E$ is a nonempty subset of $A \times D$, we have $B \subset A$ and $E \subset D$. Because $E \cap T \neq \varnothing$ we have $B \cap S=\varnothing$. Therefore, $S$ in $\mathscr{C}$ singular.

Assume now that $S$ is $\mathscr{C}$-singular. If $A \times D$ is any $(\mathscr{C} \times \mathscr{D})$ region then there is a $\mathscr{C}$-region $B \subset A$ such that $B \cap S=\varnothing$ and consequently $B \times D$ is a $(\mathscr{C} \times \mathscr{D})$-region contained in $A \times D$ with $(B \times D) \cap(S \times T)=\varnothing$. Thus, if $S$ is $\mathscr{C}$-singular then $S \times T$ is $(\mathscr{C} \times \mathscr{D}$ )-singular. Similarly, if $T$ is $\mathscr{D}$-singular then $S \times T$ is $(\mathscr{C} \times \mathscr{D})$-singular.

TheOREM 3.2. If $S$ is a $\mathscr{C}$-meager set then $S \times T$ is $(\mathscr{C} \times \mathscr{D})$ meager for any set $T \subset Y$.

Proof. Use Theorem 3.1 and the equality

$$
\left(\bigcup_{n=1}^{\infty} S_{n}\right) \times T=\bigcup_{n=1}^{\infty}\left(S_{n} \times T\right) .
$$

THEOREM 3.3. If $S$ has the Baire property with respect to $\mathscr{C}$ and $T$ has the Baire property with respect to $\mathscr{D}$, then $S \times T$ has the Baire property with repect to $\mathscr{C} \times \mathscr{D}$.

Proof. We first show $S \times Y$ has the Baire property with respect to $\mathscr{C} \times \mathscr{D}$.

Suppose $A \times D$ is any $(\mathscr{C} \times \mathscr{D})$-region. Then there is a $\mathscr{C}$-region $B \subset A$ such that $S \cap B$ or $(X-S) \cap B$ is $\mathscr{C}$-meager. The $(\mathscr{C} \times \mathscr{D})$ region $B \times D$ is contained in $A \times D$ and, by Theorem 3.2, either

$$
(S \cap B) \times D=(S \times Y) \cap(B \times D)
$$

or

$$
\begin{aligned}
{[(X-S) \cap B] \times D } & =[(X-S) \times Y] \cap(B \times D) \\
& =[(X \times Y)-(S \times Y)] \cap(B \times D)
\end{aligned}
$$

is $(\mathscr{C} \times \mathscr{D})$-meager. Hence, $S \times Y$ has the Baire property with respect to $\mathscr{C} \times \mathscr{D}$.

Similarly, $X \times T$ has the Baire property with respect to $\mathscr{C} \times \mathscr{D}$. The conclusion now follows from the equality 


$$
S \times T=(S \times Y) \cap(X \times T) .
$$

Theorem 3.4. If $(X \times Y, \mathscr{C} \times \mathscr{D})$ and $(X \times Y, \mathscr{C} \times \mathscr{E})$ are product bases and $(Y, \mathscr{D})$ is equivalent to $(Y, \mathscr{E})$ then the $(\mathscr{C} \times \mathscr{D})$ meager sets coincide with the $(\mathscr{C} \times \mathscr{E})$-meager sets.

Proof. Suppose first of all that $S$ is a subset of $X \times Y$ which is $(\mathscr{C} \times \mathscr{D})$-singular. We will show every $(\mathscr{C} \times \mathscr{E})$-region $A \times E$ contains a $(\mathscr{C} \times \mathscr{E})$-region $C \times D$ such that $(C \times D) \cap S$ is $(\mathscr{C} \times \mathscr{E})$ meager.

If $E$ is $\mathscr{E}$-meager then it follows, upon interchanging coordinates in Theorem 3.2 , that $A \times E$ is $(\mathscr{C} \times \mathscr{E})$-meager and hence so also is $(A \times E) \cap S$. Thus, we have only to consider the case where $E$ is $\mathscr{E}$-abundant.

The $\mathscr{E}$-region $E$ being $\mathscr{E}$-abundant, it follows from our hypothesis that $E$ is $\mathscr{D}$-abundant. By the Fundamental Theorem, $E$ is $\mathscr{D}$ abundant everywhere in some $\mathscr{D}$-region $B$. Since $S$ is $(\mathscr{C} \times \mathscr{D})$ singular, there is a $(\mathscr{C} \times \mathscr{D})$-region $C \times F \subset A \times B$ such that $(C \times F) \cap S=\varnothing$.

Because $E$ is $\mathscr{D}$-abundant everywhere in $B$, the $\mathscr{D}$-region $F$, which is contained in $B$, is a $\mathscr{D}$-abundant set which has the Baire property with respect to $\mathscr{D}$. Our hypothesis implies $F$ is an $\mathscr{E}$ abundant set which has the Baire property with respect to $\mathscr{E}$. Hence there is an $\mathscr{E}$-region $D$ such that $D-F$ is $\mathscr{E}$-meager. From the inclusion

$$
D \subset F \cup(D-F)
$$

we obtain

$$
C \times D \subset(C \times F) \cup[C \times(D-F)]
$$

which implies

$$
\begin{aligned}
(C \times D) & \cap S \subset[(C \times F) \cap S] \cup\{[C \times(D-F)] \cap S\} \\
= & {[C \times(D-F)] \cap S . }
\end{aligned}
$$

The set $D-F$ being $\mathscr{E}$-meager, $C \times(D-F)$ is $(\mathscr{C} \times \mathscr{E})$-meager by Theorem 3.2. Consequently $(C \times D) \cap S$ is $(\mathscr{C} \times \mathscr{E})$-meager, as we wished to show.

From the property established and the Fundamental Theorem we see that, if $S$ is $(\mathscr{C} \times \mathscr{D})$-singular then $S$ is $(\mathscr{C} \times \mathscr{E})$-meager. Therefore, if $S$ is $(\mathscr{C} \times \mathscr{D})$-meager then $S$ is a $(\mathscr{C} \times \mathscr{E})$-meager set.

Similarly, every $(\mathscr{C} \times \mathscr{E})$-meager set is $(\mathscr{C} \times \mathscr{D})$-meager. Thus, the $(\mathscr{C} \times \mathscr{D})$-meager sets are the same as the $(\mathscr{C} \times \mathscr{E})$-meager sets. 
CoRollary 3.5. If $(X, \mathscr{C})$ and $(Y, \mathscr{D})$ are topologies, $\mathscr{B}$ is a pseudo-base for $\mathscr{D}, B_{0}$ is a fixed set in $\mathscr{B}$, and

$$
\mathscr{E}=\mathscr{B} \cup\left\{B_{0} \cup(Y-\cup \mathscr{B})\right\}
$$

then the $(\mathscr{C} \times \mathscr{D})$-meager sets coincide with the $(\mathscr{C} \times \mathscr{E})$-meager sets.

TheOREM 3.6. If $(X \times Y, \mathscr{C} \times \mathscr{D})$ and $(X \times Y, \mathscr{C} \times \mathscr{E})$ are product bases satisfying CCC and $(Y, \mathscr{D})$ is equivalent to $(Y, \mathscr{E})$, then $(X \times Y, \mathscr{C} \times \mathscr{D})$ is equivalent to $(X \times Y, \mathscr{C} \times \mathscr{E})$.

Proof. Using the equivalence of $(Y, \mathscr{D})$ and $(Y, \mathscr{E})$, together with Theorem 3.3, we see that every $(\mathscr{C} \times \mathscr{D})$-region has the Baire property with respect to $\mathscr{C} \times \mathscr{C}$ and every $(\mathscr{C} \times \mathscr{E})$-region has the Baire property with respect to $\mathscr{C} \times \mathscr{D}$. The conclusion now follows from Theorems 1.6, 1.8, and 3.4.

\section{Separable bases.}

Definition. Let $(X, \mathscr{C})$ be a category base. $A$ family $\mathscr{B}$ of $\mathscr{C}$-regions with the property that each abundant set is abundant everywhere in at least one region in $\mathscr{B}$ is called a quasi-base. A category base is called separable if it has a countable quasi-base.

A particular instance of a quasi-base is the topological notion of a pseudo-base.

ExAmple 4A. Let $X$ denote the real line with the usual topology $\mathscr{C}$ and let

$$
\mathscr{D}=\{G-N: G \in \mathscr{C} \text { and } N \text { is countable }\} .
$$

The family $\mathscr{D}$ is a topology which is equivalent to $\mathscr{C}$ and $\mathscr{C}$ has a countable pseudo-base consisting of all open intervals with rational endpoints. However, $\mathscr{D}$ does not itself have a countable pseudo-base. For, suppose

$$
\mathscr{B}=\left\{G_{k}-N_{k}: k \in I\right\}
$$

is any nonempty, countable subfamily of $\mathscr{D}$-regions. If we choose a point $p_{k} \in G_{k}-N_{k}$ for each $k \in I$, then the nonempty $\mathscr{D}$-open set $X-\left\{p_{k}: k \in I\right\}$ contains none of the sets in $\mathscr{B}$.

Although the family of all open intervals with rational endpoints is not a pseudo-base for $(X, \mathscr{D})$, it is a countable quasi-base for $(X, \mathscr{D})$.

EXAMPLE 4B. Let $\mathfrak{m}$ denote an uncountable cardinal number, 
let $\theta$ be the smallest ordinal number of power $\mathfrak{m}$, and let $X$ denote the set of all ordinal numbers $\alpha<\theta$. The family $\mathscr{C}$ consisting of $\phi, X$, and all open rays $\{\beta \in X: \beta>\alpha\}$, where $\alpha \in X$, is a topology in which the singular sets coincide with the sets $S$ of power $<\mathfrak{m}$. In the case that $\mathfrak{m}$ is a regular cardinal number, every meager set is a singular set and, although $(X, \mathscr{C})$ has no countable pseudo-base, $\mathscr{B}=\{X\}$ is a countable quasi-base for $\mathscr{C}$.

ExAmply 4C. Let $X$ be an uncountable set and let $\mathscr{C}$ be the cofinite topology consisting of $\phi, X$, and all complements of finite subsets of $X$. In the case that $X$ is the set of all countable ordinal numbers, the cofinite topology is equivalent to the topology in Example 4B.

As is easily seen, $\mathscr{B}=\{X\}$ is a countable quasi-base for $(X, \mathscr{C})$. However, not only does $(X, \mathscr{C})$ have no countable pseudo-base, but it also has no equivalent, countable pseudo-base.

For, suppose

$$
\mathscr{B}=\left\{B_{k}: k \in I\right\}
$$

is any nonempty, countable family of subsets of $X$ such that $\mathscr{B}$ is a pseudo-base which is equivalent to $(X, \mathscr{C})$. Setting $\mathscr{E}=\mathscr{B} \cup\{X\}$, we have $(X, \mathscr{E})$ is equivalent to $(X, \mathscr{C})$. This equivalence, in conjunction with Corollary 1.9 , implies every set in $\mathscr{E}$ is either countable or its complement is countable. In addition, the $\mathscr{E}$-meager sets coincide with the countable subsets of $X$.

Let

$$
\mathscr{B}_{0}=\{B \in \mathscr{C}: X-B \text { is countable }\} .
$$

The family $\mathscr{B}_{0}$ is clearly nonempty and $\cap \mathscr{B}_{0}$ is the complement of a countable set. Choose a point $x_{0} \in \bigcap \mathscr{B}_{0}$. The singleton set $\left\{x_{0}\right\}$ is obviously an $\mathscr{E}$-singular set. Consequently, for each $\mathscr{E}$-region $B_{k} \in \mathscr{B}_{0}$ there is an $\mathscr{E}$-region $C_{k} \in \mathscr{B}$ such that $C_{k} \subset B_{k}$ and $x_{0} \notin C_{k}$. Each of the sets $C_{k}$ does not belong to $\mathscr{B}_{0}$ and accordingly must be countable.

Now, the set $S=X-\left\{x_{0}\right\}$ is $\mathscr{E}$-abundant. Applying the Fundamental Theorem, $S$ is $\mathscr{E}$-abundant everywhere in some $\mathscr{E}$-region $B_{k_{0}} \in \mathscr{B} . \quad$ As $B_{k_{0}}$ is thus $\mathscr{E}$-abundant, we have $B_{k_{0}} \in \mathscr{B}_{0}$. The $\mathscr{E}$ region $C_{k_{0}}$ being a subregion of $B_{k_{0}}$, the set $S \cap C_{k_{0}}$ is $\mathscr{E}$-abundant. But this is impossible, since $C_{k_{0}}$ is an $\mathscr{E}$-meager set.

REMARK. It is clear from these examples that, even in the setting of topological spaces, the theorems in the next section are proper extensions of results obtained by Kuratowski, Ulam, Sikorski, and Oxtoby. 
THEOREM 4.1. If $(X, \mathscr{C})$ is separable then the family of all abundant regions satisfies CCC.

Proof. Let $\mathscr{B}$ be a countable quasi-base for $(X, \mathscr{C})$ and assume to the contrary that there are uncountably many disjoint abundant regions. Then there exists a region $B \in \mathscr{B}$ and two disjoint abundant regions $A$ and $C$ each of which is abundant everywhere in $B$. According to Theorem 1.1, there is a region $D \subset A \cap B$. Since $C$ is abundant everywhere in $B$ and $D$ is a subregion of $B$, the set $C \cap D$ must be abundant. But $C \cap D=\varnothing$ is a meager set. Thus, we have reached a contradiction.

Definition. A category base $(X, \mathscr{C})$ is locally separable if for every point $x \in X$, every region $A$ containing $x$ has a subregion $B$ containining $x$ such that $\left(B, \mathscr{C}_{B}\right)$ is a separable category base.

Oxtoby has defined a pseudo-base $\mathscr{B}$ to be locally countable if each member of $\mathscr{B}$ contains only countably many members of $\mathscr{B}$. If $(Y, \mathscr{D})$ is a topology which has a locally countable pseudo-base $\mathscr{B}$ then $\left(Y, \mathscr{B} \cup\left\{B_{0} \cup(Y-\cup \mathscr{B})\right\}\right)$, where $B_{0}$ is a fixed element of $\mathscr{B}$, is a locally separable category base which is equivalent to $(Y, \mathscr{D})$.

THEOREM 4.2. Every separable category base is locally separable.

Proof. Let $(X, \mathscr{C})$ be a category base which has a countable quasi-base $\mathscr{B}$. It will suffice to show that for any $\mathscr{C}$-region $A$, the category base $\left(A, \mathscr{C}_{A}\right)$ is separable.

If $A$ is $\mathscr{C}$-meager then every subset of $A$ is $\mathscr{C}_{A}$-meager and the empty family is a countable quasi-base for $\mathscr{C}_{A}$. Assume therefore that $A$ is $\mathscr{C}$-abundant.

The family

$$
\mathscr{F}=\{B \in \mathscr{B}: A \cap B \text { is } \mathscr{C} \text {-abundant }\}
$$

is a nonempty, countable family of $\mathscr{C}$-regions. Applying Theorem 1.1 , for each $B \in \mathscr{F}$, we choose a $\mathscr{C}$-region $C \subset A \cap B$. The countable family $\mathscr{A}$ of $\mathscr{C}$-regions $C$ thus chosen will be a quasi-base for $\mathscr{C}_{A}$.

For, if $S$ is any $\mathscr{C}_{A}$-abundant subset of $A$ then $S$ is $\mathscr{C}$-abundant. Since $\mathscr{B}$ is a quasi-base for $(X, \mathscr{C})$, there is a $\mathscr{C}$-region $B \in \mathscr{B}$ such that $S$ is $\mathscr{C}$-abundant everwhere in $B$. The set $A \cap B$ is then $\mathscr{C}$ abundant and consequently there is a $\mathscr{C}$-region $C \in \mathscr{A}$ such that $C \subset A \cap B$. The set $S$ being $\mathscr{C}$-abundant everywhere in $B$, it is also $\mathscr{C}$-abundant everywhere in $C$. Hence, $S$ is $\mathscr{C}_{A}$-abundant everywhere in $C$. 
REMARK. The discrete topology on an uncountable set $X$ is an example of a locally separable category base which is not separable, as is also the equivalent category base $\mathscr{C}=\{\{x\}: x \in X\}$.

5. Properties involving separability. We assume throughout this section that $(X \times Y, \mathscr{C} \times \mathscr{D})$ is a given product base.

Notation. If $S \subset X \times Y$ and $x \in X$ then the section of $S$ determined by $x$, denoted by $S_{x}$, is defined by

$$
S_{x}=\{y \in Y:(x, y) \in S\} .
$$

Theorem 5.1. Assume $(Y, \mathscr{D})$ is separable. If $S$ is a $(\mathscr{C} \times \mathscr{D})$ meager set then there is a $\mathscr{C}^{\prime}$-meager set $M$ such that $S_{x}$ is $\mathscr{D}$-meager for all $x \in X-M$. Restated, almost every section of a meager set is meager.

Proof. Let $\mathscr{B}$ be a countable quasi-base for $\mathscr{D}$. We shall first consider the special case that $S$ is $(\mathscr{C} \times \mathscr{D})$-singular.

Let

$$
M=\left\{x \in X: S_{x} \text { is } \mathscr{D} \text {-abundant }\right\}
$$

and assume to the contrary that $M$ is $\mathscr{C}$-abundant. Since $\mathscr{B}$ is countable, there is a set $B \in \mathscr{B}$ and a $\mathscr{C}$-abundant set $N \subset M$ such that, for every $x \in N$, the set $S_{x}$ is $\mathscr{D}$-abundant everywhere in $B$. By the Fundamental Theorem, $N$ is $\mathscr{C}$-abundant everywhere in some $\mathscr{C}$-region $A$. The set $A \times B$ is then a $(\mathscr{C} \times \mathscr{D})$-region.

Suppose now that $C \times D$ is any $(\mathscr{C} \times \mathscr{D})$-region contained in $A \times B$. Since $N$ is $\mathscr{C}$-abundant everywhere in $A$, the set $N \cap C$ is $\mathscr{C}$-abundant. Choose a point $x \in N \cap C$. Then $S_{x}$ is $\mathscr{D}$-abundant in $D$. Choose a point $y \in S_{x} \cap D$. We then have $(x, y) \in(C \times D) \cap S$. Thus, for every $(\mathscr{C} \times \mathscr{D})$-region $C \times D \subset A \times B$ we have $(C \times \mathscr{D}) \cap$ $S \neq \varnothing$. But this implies $S$ is not $(\mathscr{C} \times \mathscr{D})$-singular. Therefore, $M$ must be a $\mathscr{C}$-meager set and the theorem is verified in the case that $S$ is $(\mathscr{C} \times \mathscr{D})$-singular.

The validity of the theorem in the general case that $S$ is $(\mathscr{C} \times \mathscr{D})$ meager is a simple consequence of this specical case and the equality

$$
\left(\bigcup_{n=1}^{\infty} S_{n}\right)_{x}=\bigcup_{n=1}^{\infty}\left(S_{n}\right)_{x}
$$

where $\left\langle S_{n}\right\rangle_{n=1}^{\infty}$ is a sequence of $(\mathscr{C} \times \mathscr{D})$-singular sets.

REMARK. Using Theorem 3.4, one sees that Theorem 5.1 remains true if the assumption that $(Y, \mathscr{D})$ is separable is replaced by the 
assumption that $(Y, \mathscr{D})$ is equivalent to a category base $(Y, \mathscr{E})$ which is separable.

As a partial converse of Theorem 5.1 we have the following generalization of a theorem proved by Kuratowski and Sierpiński in [2].

TheOREM 5.2. Assume $(Y, \mathscr{D})$ is separable. If $S$ has the Baire property with respect to $\mathscr{C} \times \mathscr{D}$ and there is a $\mathscr{C}$-meager set $P$ such that $S_{x}$ is $\mathscr{D}$-meager for all $x \in X-P$ then $S$ is $(\mathscr{C} \times \mathscr{D})$ meager.

Proof. Suppose $S$ has the Baire property with respect to $\mathscr{C} \times \mathscr{D}$ and assume to the contrary that $S$ is $(\mathscr{C} \times \mathscr{D})$-abundant. By the Fundamental Theorem, $S$ is $(\mathscr{C} \times \mathscr{D})$-abundant everywhere in a ( $\mathscr{C} \times \mathscr{D}$ )-region $A \times B$. Since $A \times B$ is $(\mathscr{C} \times \mathscr{D})$-abundant, it follows from Theorem 3.2 that $A$ is $\mathscr{C}$-abundant and $B$ is $\mathscr{D}$-abundant.

According to Theorem 1.7, the set $T=(A \times B)-S$ is $(\mathscr{C} \times \mathscr{D})$ meager. Applying Theorem 5.1, there is a $\mathscr{C}$-meager set $M$ such that $T_{x}$ is $\mathscr{D}$-meager for all $x \in X-M$. The set $A$ being $\mathscr{C}$-abundant, $T_{x}$ is $\mathscr{D}$-meager for all $x$ belonging to the $\mathscr{C}$-abundant set $A-M$. From the inclusion $(A \times B)-T \subset S$ we obtain $B-T_{x} \subset S_{x}$ for all $x \in A-M$. As $B$ is $\mathscr{D}$-abundant, the set $S_{x}$ is $\mathscr{D}$-abundant for all $x \in A-M$. This however contradicts the hypothesized existence of the $\mathscr{C}$-meager set $P$.

REMARK. This theorem is not valid for arbitrary sets $S$. For example, let $X$ denote the set of all countable ordinal numbers with the usual well-ordering and let $\mathscr{C}$ be the topology consisting of $\phi, X$, and all open rays $\{\beta \in X: \beta>\alpha\}$, where $\alpha \in X$. Then $(X \times X, \mathscr{C} \times \mathscr{C})$ is a product base in which every $(\mathscr{C} \times \mathscr{C})$-meager set is $(\mathscr{C} \times \mathscr{C})$ singular. If

$$
S=\{(\alpha, \alpha): \alpha \in X\}
$$

is the diagonal in $X \times X$ then every section of $S$ is $\mathscr{C}$-singular, but $S$ is not $(\mathscr{C} \times \mathscr{C})$-meager.

THEOREM 5.3. Assume at least one of the bases $(X, \mathscr{C})$ and $(Y, \mathscr{D})$ is locally separable. If $S \subset X$ and $T \subset Y$ then $S \times T$ is $(\mathscr{C} \times \mathscr{D})$-meager if and only if $S$ is $\mathscr{C}$-meager or $T$ is $\mathscr{D}$-meager.

Proof. Suppose $(Y, \mathscr{D})$ is locally separable, $S \times T$ is $(\mathscr{C} \times \mathscr{D})$ meager, and $S$ is $\mathscr{C}$-abundant. 
Let $y$ be any point in $Y$ and let $D$ be any $\mathscr{D}$-region containing $y$. Then there is a $\mathscr{D}$-region $E \subset D$ such that $y \in E$ and $\left(E, \mathscr{D}_{E}\right)$ is separable. The set $S \times(T \cap E)$ is $(\mathscr{C} \times \mathscr{D})$-meager and, as is easily verified, it is also $\left(\mathscr{C} \times \mathscr{D}_{E}\right)$-meager. By Theorem 5.1, there is a point $x \in S$ such that

$$
[S \times(T \cap E)]_{x}=T \cap E
$$

is a $\mathscr{D}_{E}$-meager set. Consequently, $T \cap E$ is $\mathscr{D}$-meager. From Theorem 1.5 we see that $T$ is $\mathscr{D}$-meager. Thus, if $S \times T$ is $(\mathscr{C} \times \mathscr{D})$ meager then either $S$ is $\mathscr{C}$-meager or $T$ is $\mathscr{D}$-meager.

As established in Theorem 3.2, the converse is also true.

Using Corollary 3.5 we obtain the following consequence of Theorem 5.3 which was established by Oxtoby.

CoRollary 5.4. If $(X, \mathscr{C})$ and $(Y, \mathscr{D})$ are topologies, at least one of which has a locally countable pseudo-base, and $S \subset X, T \subset Y$, then $S \times T$ is of the first category in $X \times Y$ if and only if $S$ is of the first category in $X$ or $T$ is of the first category in $Y$.

THEOREM 5.5. If both $(X, \mathscr{C})$ and $(Y, \mathscr{D})$ are separable then $(X \times Y, \mathscr{C} \times \mathscr{D})$ is also separable.

Proof. Let $\mathscr{A}$ and $\mathscr{B}$ be countable quasi-bases for $\mathscr{C}$ and $\mathscr{D}$, respectively. We will show the countable family $\mathscr{E}=\mathscr{A} \times \mathscr{B}$ is a quasi-base for $\mathscr{C} \times \mathscr{D}$.

Suppose $S$ is a $(\mathscr{C} \times \mathscr{D})$-abundant set. By the Fundamental Theorem, $S$ is $(\mathscr{C} \times \mathscr{D})$-abundant everywhere in some $(\mathscr{C} \times \mathscr{D})$ region $C \times D$. From Theorem 3.2 we see that $C$ is $\mathscr{C}$-abundant and $D$ is $\mathscr{D}$-abundant. Let $C$ be $\mathscr{C}$-abundant everywhere in a region $A \in \mathscr{A}$ and let $D$ be $\mathscr{D}$-abundant everywhere in a region $B \in \mathscr{B}$. Using Theorems 4.2 and 5.3 we see that $C \times D$ is $(\mathscr{C} \times \mathscr{D})$-abundant everywhere in the region $A \times B \in \mathscr{E}$. By Theorem 1.3, $S$ is $(\mathscr{C} \times \mathscr{D})$ abundant everywhere in $A \times B$.

From this theorem it is a simple matter to derive the following.

THEOREM 5.6. If both $(X, \mathscr{C})$ and $(Y, \mathscr{D})$ are locally separable then $(X \times Y, \mathscr{C} \times \mathscr{D})$ is locally separable.

THEOREM 5.7. Assume at least one of the bases $(X, \mathscr{C})$ and $(Y, \mathscr{D})$ is locally separable. If $S \subset X$ and $T \subset Y$ then $S \times T$ has the Baire property with respect to $\mathscr{C} \times \mathscr{D}$ if and only if either both $S$ and $T$ have the Baire property or one of them is meager. 
Proof. Assume $S \times T$ has the Baire property with respect to $\mathscr{C} \times \mathscr{D}, S$ is $\mathscr{C}$-abundant, and $T$ is $\mathscr{D}$-abundant. We have to show $S$ has the Baire property with respect to $\mathscr{C}$ and $T$ has the Baire property with respect to $\mathscr{D}$.

Let $A$ be any $\mathscr{C}$-region in which $S$ is $\mathscr{C}$-abundant everywhere and let $B$ be any $\mathscr{D}$-region in which $T$ is $\mathscr{D}$-abundant everywhere. By means of Theorem 5.3 we see that $S \times T$ is $(\mathscr{C} \times \mathscr{D})$-abundant everywhere in the $(\mathscr{C} \times \mathscr{D})$-region $A \times B$. Since $S \times T$ has the Baire property, the set

$$
[(X \times Y)-(S \times T)] \cap(A \times B)
$$

is $(\mathscr{C} \times \mathscr{D})$-meager. The two subsets

$$
[(X-S) \times T] \cap(A \times B)=[(X-S) \cap A] \times(T \cap B)
$$

and

$$
[S \times(Y-T)] \cap(A \times B)=(S \cap A) \times[(Y-T) \cap B]
$$

of this set are then also $(\mathscr{C} \times \mathscr{D})$-meager. Since $T \cap B$ is $\mathscr{D}$ abundant and $S \cap A$ is $\mathscr{C}$-abundant, it follows from Theorem 5.3 that $(X-S) \cap A$ is $\mathscr{C}$-meager and $(Y-T) \cap B$ is $\mathscr{D}$-meager. Therefore, $S$ has the Baire property with respect to $\mathscr{C}$ and $T$ has the Baire property with respect to $\mathscr{D}$.

We thus see that if $S \times T$ has the Baire property with respect to $\mathscr{C} \times \mathscr{D}$ then either both $S$ and $T$ have the Baire property or one of the sets $S$ and $T$ is meager. The converse of this is a consequence of Theorems 3.2 and 3.3 .

Theorem 5.8. Assume $(Y, \mathscr{D})$ is separable. If $S$ has the Baire property with respect to $\mathscr{C} \times \mathscr{D}$ then there is a $\mathscr{C}$-meager set $M$ such that $S_{x}$ has the Baire property with respect to $\mathscr{D}$ for all $x \in X-M$. Restated, almost every section of a set having the Baire property has the Baire property.

Proof. Let $\mathscr{B}$ be a countable quasi-base for $\mathscr{D}$. Assume to the contrary that the set

$$
M=\left\{x \in X: S_{x} \notin \mathfrak{B}(\mathscr{D})\right\}
$$

is a $\mathscr{C}$-abundant set. For each $x \in M$, the sets $S_{x}$ and $Y-S_{x}$ are then $\mathscr{D}$-abundant everywhere in some $\mathscr{D}$-region. From the definition of a quasi-base and Theorem 1.3, both $S_{x}$ and $Y-S_{x}$ are $\mathscr{D}$-abundant everywhere in some $\mathscr{D}$-region $B \in \mathscr{B}$. Since $\mathscr{B}$ is countable, there is a $\mathscr{D}$-region $B \in \mathscr{B}$ and a $\mathscr{C}$-abundant set $N \subset M$ such that, for every $x \in N$, both $S_{x}$ and $Y-S_{x}$ are $\mathscr{D}$-abundant everywhere in $B$. According to the Fundamental Theorem, $N$ is $\mathscr{C}$-abundant everywhere 
in some $\mathscr{C}$-region $A$. We shall show both $S$ and $(X \times Y)-S$ are $(\mathscr{C} \times \mathscr{D})$-abundant everywhere in the $(\mathscr{C} \times \mathscr{D})$-region $A \times B$, which contradicts our assumption that $S$ has the Baire property with respect to $\mathscr{C} \times \mathscr{D}$.

Suppose $C \times D$ is any $\left(\mathscr{C}^{\circ} \times \mathscr{D}\right)$-region contained in $A \times B$, then $C \subset A$ and $D \subset B$. Hence $C \cap N$ is $\mathscr{C}$-abundant and for every $x \in$ $C \cap N$ both $D \cap S_{x}$ and $D \cap\left(Y-S_{x}\right)$ are $\mathscr{D}$-abundant sets. By Theorem 5.1, the sets $(C \times D) \cap S$ and $(C \times D) \cap[(X \times Y)-S]$ are both $(\mathscr{C} \times \mathscr{D})$-abundant. Thus we have reached the desired contradiction.

Definition. A category base $(X, \mathscr{C})$ is called a Baire base if every region is an abundant set.

From Theorem 5.3 we easily derive the following.

THEOREM 5.9. If $(X, \mathscr{C})$ and $(Y, \mathscr{D})$ are Baire bases, at least one of which is locally separable, then $(X \times Y, \mathscr{C} \times \mathscr{D})$ is a Baire base.

Notation. If $f: X \rightarrow Y$ is a function then the graph of $f$ will be denoted by $\Gamma(f)$; i.e.

$$
\Gamma(f)=\{(x, y) \in X \times Y: y=f(x)\} .
$$

Theorem 5.10, Assume $\left(X, \mathscr{C}^{\circ}\right)$ is a Baire base and $(Y, \mathscr{D})$ is a locally separable Baire base such that each singleton subset of $Y$ is $\mathscr{D}$-meager. If $f: X \rightarrow Y$ is any function then the complement of the graph of $f$ is $(\mathscr{C} \times \mathscr{D})$-abundant at each of its points.

Proof. Let $T=(X \times Y)-\Gamma(f)$ and assume to the contrary that there is a point $z \in T$ at which $T$ is $(\mathscr{C} \times \mathscr{D})$-meager. Then there exists a $(\mathscr{C} \times \mathscr{D})$-region $C \times D$ such that $z \in C \times D$ and the set $T \cap(C \times D)$ is $(\mathscr{C} \times \mathscr{D})$-meager. From the definition of local separability, there is a $\mathscr{D}$-region $E \subset D$ such that $z \in C \times E$ and $\left(E, \mathscr{D}_{E}\right)$ is separable. According to Theorem $2.3,\left(X \times E, \mathscr{C} \times \mathscr{D}_{E}\right)$ is a product base.

The set $S=T \cap(C \times E)$ being a $(\mathscr{C} \times \mathscr{D})$-meager subset of $C \times E$, it is also $\left(\mathscr{C} \times \mathscr{D}_{E}\right)$-meager. Applying Theorem 5.1, there is a $\mathscr{C}$-meager set $M$ such that $S_{x}$ is $\mathscr{D}_{E}$-meager for all $x \in X-M$. Since $C$ is $\mathscr{C}$-abundant, there is a point $x \in C-M$ and

$$
S_{x}=[T \cap(C \times E)]_{x}=T_{x} \cap E
$$

is $\mathscr{D}_{E}$-meager. Now, $T$ being the complement of the graph of $f$, 
the set $T_{x}$ differs from $Y$ by only a single point; namely, the point $(x, f(x))$. Consequently, $T_{x}$ is a $\mathscr{D}$-comeager set. As $T_{x} \cap E$ is $\mathscr{D}_{E^{-}}$ meager, hence also $\mathscr{D}$-meager, and $T_{x}$ is $\mathscr{D}$-comeager, $E$ must be a $\mathscr{D}$-meager set. But this contradicts the hypothesis that $(Y, \mathscr{D})$ is a Baire base.

THEOREM 5.11. Assume $(Y, \mathscr{D})$ is a locally separable base such that each singleton subset of $Y$ is $\mathscr{D}$-meager. If $f: X \rightarrow Y$ is a function whose graph $\Gamma(f)$ has the Baire property with respect to $\mathscr{C} \times \mathscr{D}$ then $\Gamma(f)$ is a $(\mathscr{C} \times \mathscr{D})$-meager set.

Proof. Let $z$ be any point of $X \times Y$ and let $A \times B$ be any $(\mathscr{C} \times \mathscr{D}$ )-region containing $z$. Then there is a $\mathscr{D}$-region $E \subset B$ such that $z \in A \times E$ and $\left(E, \mathscr{D}_{E}\right)$ is separable. According to Theorem 2.3, $\left(X \times E, \mathscr{C} \times \mathscr{D}_{E}\right)$ is a product base.

Since $\Gamma(f)$ has the Baire property with respect to $\mathscr{C} \times \mathscr{D}$, the set

$$
S=\Gamma(f) \cap(A \times E)
$$

has the Baire property with respect to $\mathscr{C} \times \mathscr{D}$. Hence, $S$ has the Baire property with respect to $\mathscr{C} \times \mathscr{D}_{E}$. For every point $x \in X$,

$$
S_{x}=[\Gamma(F)]_{x} \cap(A \times E)_{x}
$$

consists of at most one point and hence is $\mathscr{D}_{E}$-meager. Applying Theorem 5.2, we see that $S$ is $\left(\mathscr{C} \times \mathscr{D}_{E}\right)$-meager. Consequently, $S$ is $(\mathscr{C} \times \mathscr{D})$-meager.

We have thus shown that if $z$ is any point of $X \times Y$ then every $(\mathscr{C} \times \mathscr{D})$-region containing $z$ has a $(\mathscr{C} \times \mathscr{D})$-subregion containing $z$ in which $\Gamma(f)$ is $(\mathscr{C} \times \mathscr{D})$-meager. We conclude from Theorem 1.5 that $\Gamma(f)$ is a $(\mathscr{C} \times \mathscr{D})$-meager set.

\section{REFERENCES}

1. P. Erdös and J. Oxtoby, Partitions of the plane into sets having positive measure in every non-null measurable product set, Trans. Amer. Math. Soc., 79 (1955), 91-102. 2. C. Kuratowski and W. Sierpiński, Sur l'existence des ensembles projectifs non mesurables, Bulgarska Akademiia na Naukite. Spisanie. Kniga 2: Klon Prirodo. Matematicken, 61 (1941), 207-212.

3. C. Kuratowski and S. Ulam, Quelques propriétés topologiques du produit combinatoire, Fundamenta Mathematicae, 19 (1932), 247-251.

4. J. C. Morgan II, Infinite games and singular sets, Colloquium Mathematicum, 29 (1974), 7-17.

5. - Baire category from an abstract viewpoint, Fundamenta Mathematicae, 94 (1977), 13-23.

6. - On translation invariant families of sets, Colloquium Mathematicum, 34 (1975), 63-68.

7. — On zero-one laws, Proc. Amer. Math. Soc., 62 (1977), 353-358. 
8. J. C. Morgan II, The absolute Baire property, Pacific J. Math., 65 (1976), 421-436. 9. - On the absolute Baire property, ibid., 78 (1978), 415-431.

10. J. Oxtoby, Cartesian products of Baire spaces, Fundamenta Mathematicae, 49 (1961), 157-166.

11. R. Sikorski, On the cartesian product of metric spaces, ibid., 34 (1947), 288-292. 12. E. Szpilrajn (Marczewski), Sur une classe de fonctions de M. Sierpiński et la classe correspondante d'ensembles, ibid., 24 (1935), 17-34.

Received June 24, 1981.

California State Polytechnic University

Pomona, CA 91768 


\section{PACIFIC JOURNAL OF MATHEMATICS}

\section{EDITORS}

DONALD BABBITT (Managing Editor)

University of California

Los Angeles, California 90024

\section{Hugo Rossi}

University of Utah

Salt Lake City, UT 84112

C. C. MOore and Arthur AGuS

University of California

Berkeley, CA 94720
J. DugundJI

Department of Mathematics University of Southern California Los Angeles, California 90007

R. FinN and J. Milgram Stanford University Stanford, California 94305

ASSOCIATE EDITORS
R. ARENS
E. F. BECKENBACH
B. H. NeumanN
F. WOLF
K. YOSHIDA

\section{SUPPORTING INSTITUTIONS}

UNIVERSITY OF ARIZONA

UNIVERSITY OF BRITISH COLUMBIA

CALIFORNIA INSTITUTE OF TECHNOLOGY

UNIVERSITY OF CALIFORNIA

MONTANA STATE UNIVERSITY

UNIVERSITY OF NEVADA, RENO

NEW MEXICO STATE UNIVERSITY

OREGON STATE UNIVERSITY
UNIVERSITY OF OREGON

UNIVERSITY OF SOUTHERN CALIFORNIA

STANFORD UNIVERSITY

UNIVERSITY OF HAWAII

UNIVERSITY OF TOKYO

UNIVERSITY OF UTAH

WASHINGTON STATE UNIVERSITY

UNIVERSITY OF WASHINGTON 


\section{Pacific Journal of Mathematics}

Vol. 99, No. $1 \quad$ May, 1982

Mariano Giaquinta, Jindrich Necas, O. John and J. Stará, On the

regularity up to the boundary for second order nonlinear elliptic systems . . 1

Siegfried Graf, Realizing automorphisms of quotients of product $\sigma$-fields . . 19

Alfred Washington Hales and Ernst Gabor Straus, Projective colorings . . . 31

Sandra Hayes, The weak Nullstellensatz for finite-dimensional complex

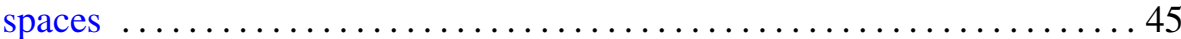

Gerald Norman Hile and Murray Harold Protter, The Cauchy problem

and asymptotic decay for solutions of differential inequalities in Hilbert

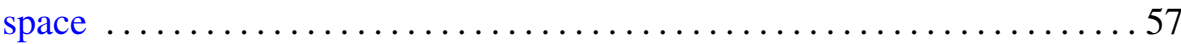

Robert D. Little, Projective space as a branched covering with orientable

branch set ......................................... 89

Jaroslav Mach, On the proximinality of Stone-Weierstrass subspaces . . . . . 997

John C. Morgan, II, On product bases ...................... 105

K. Balakrishna Reddy and P. V. Subrahmanyam, Altman's contractors

and fixed points of multivalued mappings . .................. 127

James Ted Rogers Jr., Decompositions of homogeneous continua . . . . . . . 137

Ahmed Ramzy Sourour, Characterization and order properties of

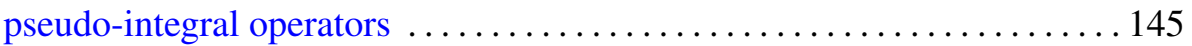

Robert Moffatt Stephenson Jr., Pseudocompact and Stone-Weierstrass

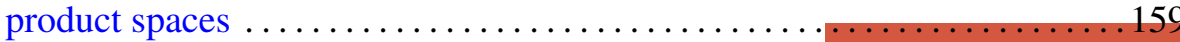

Bruce Stewart Trace, On attaching 3-handles to a 1-connected

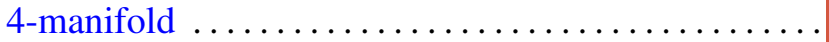

Akihito Uchiyama, The construction of certain BMO functions and the corona problem

Thomas Alva Whitehurst, An application of orthogonal polynomials to random walks ..............................

David J. Winter, Root locologies and idempotents of Lie and nonassociative algebras

William Robin Zame, The classification of uniform algebras on plane domains 